\title{
ON THE EXPONENTS MODULO 3 IN THE STANDARD FACTORISATION OF $n$ !
}

\author{
Wei Liu And Yong-Gao Chen
}

Let $p$ be a prime and $m$ be a positive integer. For a positive integer $n$, let $e_{p}(n)$ be the nonnegative integer with $p^{e_{p}(n)} \mid n$ and $p^{e_{p}(n)+1} \nmid n$. As a corollary of our main result we derive an asymptotic formula for the counting function with regard to the condition $e_{p}(n !) \equiv \varepsilon(\bmod 3)$, where $\varepsilon \in Z_{3}$. In 2001 , Sander proved the result with modulus 2 .

\section{INTRODUCTION}

Let $p_{1}, p_{2}, \ldots$ be the sequence of all primes in ascending order. For a positive integer $n$, let $e_{p_{i}}(n)$ be the nonnegative integer with $p_{i}^{e_{p_{i}}(n)} \mid n$ and $p_{i}^{e_{p_{i}}(n)+1} \nmid n$. In 1997, Berend [1] proved a conjecture of Erdös and Graham (see [4, p. 77]) by showing that for every positive integer $k$ there exist infinitely many positive integers $n$ with

$$
e_{p_{1}}(n !) \equiv 0 \quad(\bmod 2), e_{p_{2}}(n !) \equiv 0 \quad(\bmod 2), \ldots, e_{p_{k}}(n !) \equiv 0 \quad(\bmod 2),
$$

where the differences between adjacent such $n$ are less than effectively compitable constant depending only on $k$. The initial case $n=1$ is very useful in Berend's proof.

In 1999, Chen and Zhu [3] considered a general case. Two years iater, Sander [6] posed two conjectures and proved some special cases of his conjectures. After Sander, Chen [2] proved one of the two conjectures posed by Sander: for any given positive integer $k$ and any $\varepsilon_{i} \in\{0,1\}(i=1,2, \ldots, k)$, there exist infinitely many positive integers $n$ such that

$$
e_{p_{1}}(n !) \equiv \varepsilon_{1} \quad(\bmod 2), e_{p_{2}}(n !) \equiv \varepsilon_{2} \quad(\bmod 2), \ldots, e_{p_{k}}(n !) \equiv \varepsilon_{k} \quad(\bmod 2) .
$$

In 2003, F. Luca and P. Stănică [5] posed the following conjecture:

Conjecture (F.Luca and P.Stănică [5]). Let $p_{1}, \ldots, p_{k}$ be distinct primes, $m_{1}, \ldots, m_{k}$ be arbitrary positive integers $(\geqslant 2)$, and $0 \leqslant a_{i} \leqslant m_{i}-1$ for $i=1, \ldots, k$ be arbitrary residue class modulo $m_{i}$. Then

$$
\left|\left\{0 \leqslant n<N: e_{p_{i}}(n !) \equiv a_{i} \quad\left(\bmod m_{i}\right), 1 \leqslant i \leqslant k\right\}\right| \sim \frac{N}{m_{1} \ldots m_{k}} \text { as } N \rightarrow \infty .
$$

Received 10th October, 2005

The author was supported by the National Natural Science Foundation of China, Grant No 10471064 . The authors would like to thank the referee for his/her useful comments.

Copyright Clearance Centre, Inc. Serial-fee code: 0004-9727/06 \$A2.00+0.00. 
In their paper, they proved the conjecture under the assumption that $p_{i} \nmid m_{i}(i$ $=1, \ldots, k)$.

Sander [6] derived an asymptotic formula for the proportion of $n<N$ for which $e_{p}(n !) \equiv \varepsilon(\bmod 2)$. In the present paper, we improve the method in Sander [6] and derive an asymptotic formula for the counting function with regard to the condition $e_{p}(n !) \equiv \varepsilon(\bmod 3)$, where $p$ is a prime and $\varepsilon \in \mathbf{Z}_{3}$. At the same time we prove a more general result. Although it is also a special case of Luca-Stănică Conjecture, the following theorem not only gives a good bound for the error term, but also can take any prime $p$ as modulus.

Let $m$ be a fixed positive integer. For $\varepsilon \in \mathbf{Z}_{m}$ and a prime $p$, let

$$
E_{p, \varepsilon, m}(N):=\left|\left\{0 \leqslant n<N: e_{p}(n !) \equiv \varepsilon \quad(\bmod m)\right\}\right| .
$$

Theorem. For any prime $p$ with $p \equiv \pm 1(\bmod m)$ or $p \equiv 0(\bmod m)$ and any $\varepsilon \in Z_{m}$, we have

$$
E_{p, \varepsilon, m}(N)=\frac{1}{m} N+O\left(N^{1 / 2}\right)
$$

REMARK. From the proof of the theorem, we can see that

$$
\left|E_{p, \varepsilon, m}(N)-\frac{1}{m} N\right| \leqslant 4 p^{3 / 2} N^{1 / 2} \text {. }
$$

Noting that all primes have the property that $p \equiv \pm 1(\bmod 3)$ or $p \equiv 0(\bmod 3)$, by the theorem, we get the following corollary:

COROLlary 1. For any prime $p$ and any $\varepsilon \in Z_{3}$, we have

$$
E_{p, \varepsilon, 3}(N)=\frac{1}{3} N+O\left(N^{1 / 2}\right)
$$

By setting $m=p$ in the theorem we get the corollary:

Corollary 2. Let $p$ be a prime. For any $\varepsilon \in Z_{p}$, we have

$$
E_{p, \varepsilon, p}(N)=\frac{1}{p} N+O\left(N^{1 / 2}\right)
$$

\section{PROOF OF THE THEOREM}

Let $p$ be a prime, $m$ be a positive integer and let the nonnegative integer $n$ have the $p$-adic expansion $n=n_{s} p^{s}+\cdots+n_{1} p+n_{0}$ with $p$-adic digits $0 \leqslant n_{j}<p$ for $0 \leqslant j \leqslant s$. It is well known that

$$
e_{p}(n !)=\sum_{j=1}^{\infty}\left[\frac{n}{p^{j}}\right]
$$


Hence,

$$
e_{p}(n !)=\sum_{j=1}^{s} n_{j}\left(p^{j-1}+\cdots+p+1\right) \equiv \sum_{j=1}^{s} a_{j} n_{j} \quad(\bmod m),
$$

where $a_{j} \equiv p^{j-1}+\cdots+p+1(\bmod m)$ and $0 \leqslant a_{j}<m, j=1, \ldots, s$. Now, we give two lemmas first.

Lemma 1. Let $p$ be a prime, $m$ be a positive integer, and $r \geqslant 2$ be an integer. For a fixed integer $k(1 \leqslant k \leqslant m-1)$, we have

$$
\mid\left\{j \mid 1 \leqslant j \leqslant r-1 \text { and } k a_{j} \equiv 0 \quad(\bmod m)\right\} \mid \leqslant \frac{r-1}{2},
$$

where $a_{j}(j=1, \ldots, r-1)$ are defined as above.

Proof: Suppose that $m \mid k a_{j}$ and $m \mid k a_{j+1}$ for some $j(1 \leqslant j \leqslant r-2)$. By the definition of $a_{j}(1 \leqslant j \leqslant r-1)$, we have $m \mid k p^{j}$.

Assume that $m=p^{\alpha} m_{1}$, where $p \nmid m_{1}$ and $\alpha \geqslant 0$. Since $m \mid k a_{j}$, we have $m \mid$ $k\left(1+p+\cdots+p^{j-1}\right)$. Hence, $p^{\alpha} \mid k\left(1+p+\cdots+p^{j-1}\right)$. Since $\left(p, 1+p+\cdots+p^{j-1}\right)=1$, we have $p^{\alpha} \mid k$. Then assume that $k=k_{1} p^{\alpha}$, where $k_{1}$ be an integer. From $m \mid k p^{j}$, we have that $k p^{j}=k_{1} p^{\alpha+j} \equiv 0(\bmod m)$, hence, $m_{1} \mid k_{1}$. Now, we have $m \mid k$, a contradiction with $1 \leqslant k \leqslant m-1$.

Hence, for each $j(j=1, \ldots, r-2)$, we have either $k a_{j} \not \equiv 0(\bmod m)$ or $k a_{j+1} \not \equiv 0$ $(\bmod m)$.

By $k a_{1}=k \not \equiv 0(\bmod m)$, we obtain a proof of Lemma 1 .

Lemma 2. Let $p$ be a prime, $m$ be a positive integer, $r \geqslant 0, U \geqslant 1$ and $T \geqslant 0$ be integers with $U \equiv \pm 1(\bmod m)$ or $U \equiv 0(\bmod m)$, and let $\varepsilon \in\{0,1, \ldots, m-1\}$. Then

$$
\begin{aligned}
C(\varepsilon):=\mid\left\{\left(n_{r}, \ldots, n_{0}\right) \in \mathbb{Z}^{+1}: 0 \leqslant n_{j}<U(0 \leqslant j<r) ; 0 \leqslant n_{r}<T ;\right. \\
\\
\left.\qquad \sum_{j=1}^{r} a_{j} n_{j} \equiv \varepsilon \quad(\bmod m)\right\} \mid,
\end{aligned}
$$

where $a_{j}(j=1, \ldots, r)$ are defined as above and $\sum_{j=1}^{r} a_{j} n_{j}=0$ for $r=0$, satisfies

$$
\left|C(\varepsilon)-\frac{1}{m} T U^{r}\right| \leqslant T U^{(r+1) / 2} \text {. }
$$

Proof: The result is trivial for $r=0,1$. Now we assume that $r \geqslant 2$. Let $\omega_{k}=e^{(2 \pi i k) / m}(k=0,1, \ldots, m-1)$, which are all the roots of $x^{m}=1$. Then for any integer $k(0 \leqslant k \leqslant m-1)$, we have

$$
\begin{aligned}
C(0)+\omega_{k} C(1)+\omega_{k}^{2} C(2)+\cdots+\omega_{k}^{m-1} C(m-1) & =\sum_{n_{0}=0}^{U-1} \sum_{n_{1}=0}^{U-1} \ldots \sum_{n_{r-1}=0}^{U-1} \sum_{n_{r}=0}^{T-1} \omega_{k}^{\sum_{j=1}^{r} a_{j} n_{j}} \\
& =U \prod_{j=1}^{r-1}\left(\sum_{n_{j}=0}^{U-1} \omega_{k}^{a_{j} n_{j}}\right) \sum_{n_{r}=0}^{T-1} \omega_{k}^{a_{r} n_{r}} .
\end{aligned}
$$


Let $B(\varepsilon)=C(\varepsilon)-(1 / m) T U^{r}(0 \leqslant \varepsilon<m)$, we have

$$
\begin{aligned}
& B(0)+\omega_{0} B(1)+\omega_{0}^{2} B(2)+\cdots+\omega_{0}^{m-1} B(m-1)=0 \\
& B(0)+\omega_{k} B(1)+\omega_{k}^{2} B(2)+\cdots+\omega_{k}^{m-1} B(m-1) \\
& =U \prod_{j=1}^{r-1}\left(\sum_{n_{j}=0}^{U-1} \omega_{k}^{a_{j} n_{j}}\right) \sum_{n_{r}=0}^{T-1} \omega_{k}^{a_{r} n_{r}}, 1 \leqslant k \leqslant m-1 .
\end{aligned}
$$

For any integer $u(1 \leqslant u \leqslant m)$, multiply both sides by $\omega_{k}^{u}$, then add all the equations, we have

$$
m B(m-u)=\sum_{k=1}^{m-1} \omega_{k}^{u} U \prod_{j=1}^{r-1}\left(\sum_{n_{j}=0}^{U-1} \omega_{k}^{a_{j} n_{j}}\right) \sum_{n_{r}=0}^{T-1} \omega_{k}^{a_{r} n_{r}} .
$$

For a fixed $k(1 \leqslant k \leqslant m-1)$, it follows from Lemma 1 that

$$
\mid\left\{j \mid 1 \leqslant j \leqslant r-1 \text { and } k a_{j} \equiv 0 \quad(\bmod m)\right\} \mid \leqslant \frac{r-1}{2} .
$$

If $k a_{j} \neq 0(\bmod m)$, since $U \equiv \pm 1(\bmod m)$ or $U \equiv 0(\bmod m)$, we have

$$
\left|\sum_{n_{j}=0}^{U-1} \omega_{k}^{a_{j} n_{j}}\right| \leqslant 1
$$

Then

$$
m|B(m-u)| \leqslant \sum_{k=1}^{m-1} T U^{(r-1) / 2+1} \leqslant m T U^{(r+1) / 2}
$$

hence,

$$
|B(m-u)| \leqslant T U^{(r+1) / 2}
$$

Thus for any $\varepsilon(\varepsilon=0,1, \ldots, m-1)$, we have

$$
\left|C(\varepsilon)-\frac{1}{m} T U^{r}\right| \leqslant T U^{(r+1) / 2} .
$$

This completes the proof of Lemma 2.

PROOF OF THE THEOREM: We define $E_{p, \varepsilon, m}(L, M):=E_{p, \varepsilon, m}(L)-E_{p, \epsilon, m}(M)$ for integers $L \geqslant M \geqslant 0$. Let $N=N_{s} p^{s}+\cdots+N_{1} p+N_{0}$ be the $p$-adic expansion of $N$. Originating from the disjoint union of the corresponding sets, we immediately have

$$
E_{p, \varepsilon, m}(N)=\sum_{k=0}^{s} E_{p, \varepsilon, m}\left(N_{s} p^{s}+\cdots+N_{s-k} p^{s-k}, N_{s} p^{s}+\cdots+N_{s-k+1} p^{s-k+1}\right)
$$

where the empty sum occurring for $k=0$ is considered to be 0 . 
For a fixed integer $k$, we obtain

$$
\begin{aligned}
& E_{p, \varepsilon, m}\left(N_{s} p^{s}+\cdots+N_{s-k} p^{s-k}, N_{s} p^{s}+\cdots+N_{s-k+1} p^{s-k+1}\right) \\
& =\mid\left\{n=N_{s} p^{s}+\cdots+N_{s-k+1} p^{s-k+1}+n_{s-k} p^{s-k}+\cdots+n_{1} p+n_{0}:\right. \\
& \left.0 \leqslant n_{s-k-j}<p(1 \leqslant j \leqslant s-k) ; 0 \leqslant n_{s-k}<N_{s-k} ; e_{p}(n !) \equiv \varepsilon \quad(\bmod m)\right\} \mid \\
& =\mid\left\{\left(n_{s-k}, \ldots, n_{0}\right): 0 \leqslant n_{j}<p(0 \leqslant j<s-k) ; 0 \leqslant n_{s-k}<N_{s-k} ;\right. \\
& \left.\sum_{j=1}^{s-k} a_{j} n_{j} \equiv \varepsilon-\sum_{j>s-k} a_{j} N_{j} \quad(\bmod m)\right\} \mid \text {. }
\end{aligned}
$$

It follows from Lemma 2, that

$$
\left|E_{p, \varepsilon, m}\left(N_{s} p^{s}+\cdots+N_{s-k} p^{s-k}, N_{s} p^{s}+\cdots+N_{s-k+1} p^{s-k+1}\right)-\frac{1}{m} N_{s-k} p^{s-k}\right| \leqslant N_{s-k} p^{(s-k+1) / 2} .
$$

Hence, we have

$$
\begin{aligned}
& \left|E_{p, \varepsilon, m}(N)-\frac{1}{m} N\right| \\
& \quad \leqslant \sum_{k=0}^{s}\left|E_{p, \varepsilon, m}\left(N_{s} p^{s}+\cdots+N_{s-k} p^{s-k}, N_{s} p^{s}+\cdots+N_{s-k+1} p^{s-k+1}\right)-\frac{1}{m} N_{s-k} p^{s-k}\right| \\
& \quad \leqslant \sum_{k=0}^{s} N_{s-k} p^{(s-k+1) / 2} .
\end{aligned}
$$

Since $N_{s-k}<p, p \geqslant 2$ and $p^{s} \leqslant N$, we have

$$
\sum_{k=0}^{s} N_{s-k} p^{(s-k+1) / 2}=\sum_{k=0}^{s} p^{(s-k+3) / 2}=p^{(s+3) / 2} \sum_{k=0}^{s} p^{-(k / 2)} \leqslant 4 p^{3 / 2} N^{1 / 2} .
$$

From it, we have $E_{p, \varepsilon, m}(N)=(1 / m) N+O\left(N^{1 / 2}\right)$. This completes the proof of the theorem.

\section{REFERENCES}

[1] D. Berend, 'On the parity of exponents in the factorization of $n$ !', J. Number Theory 64 (1997), 13-19.

[2] Y.G. Chen, 'On the parity of exponents in the standard factorization of n!', J. Number Theory 100 (2003), 326-331.

[3] Y.G. Chen and Y.C. Zhu, 'On the prime power factorization of n!', J. Number Theory $82(2000), 1-11$.

[4] P. Erdös and R.L. Graham, Old and new problems and results in combinatorial number theory (L'Enseignement Mathématique, Imprimerie Kundig, Geneva, 1980).

[5] F. Luca and P. Stănică, 'On the prime power factorization of $n$ !', J. Number Theory 102 (2003), 298-305. 
[6] J.W. Sander, 'On the parity of exponents in the prime factorization of factorials', $J$. Number Theory 90 (2001), 316-328.

Department of Mathematics

Nanjing Normal University

Nanjing 210097

China

e-mail:ygchen@njnu.edu.cn 